\title{
Malignant Peripheral Nerve Sheath Tumor in the Lesser Sac Masquerading as a Gastrointestinal Stromal Tumor of the Stomach: A Case Report
}

\author{
Seung-Gu Yeo, ${ }^{1}$ Eun Seok Kim, ${ }^{1}$ Min-Jeong Kim,,${ }^{2}{ }^{*}$ Hong Il Ha, ${ }^{2}$ Kwanseop Lee, ${ }^{2}$ and Mi Jung Kwon ${ }^{3}$ \\ ${ }^{1}$ Department of Radiation Oncology, Soonchunhyang University College of Medicine, Soonchunhyang University Hospital, Cheonan, Republic of Korea \\ ${ }^{2}$ Department of Radiology, Hallym University College of Medicine, Hallym University Sacred Heart Hospital, Anyang, Republic of Korea \\ ${ }^{3}$ Department of Pathology, Hallym University College of Medicine, Hallym University Sacred Heart Hospital, Anyang, Republic of Korea \\ "Corresponding author: Min-Jeong Kim, Department of Radiology, Hallym University Sacred Heart Hospital, Anyang, Republic of Korea. Tel: +82-313803918, Fax: +82-313803878, \\ E-mail: drkmj@hallym.or.kr
}

Received 2016 June 04; Accepted 2016 August 05.

\begin{abstract}
A malignant peripheral nerve sheath tumor (MPNST) is very rare, representing $3-10 \%$ of all soft tissue sarcomas. Approximately half of all such tumors are diagnosed in patients with neurofibromatosis type 1 (NF1). The extremities are involved most commonly, and the patient's age is usually 20 - 50 years. Herein, we present a case of an MPNST that developed sporadically in an unusual location and in an unusually younger patient. A 16-year-old girl presented with dyspepsia and abdominal distension. Computed tomography (CT) demonstrated a well-defined mass approximately $12 \mathrm{~cm}$ in diameter in the lesser sac. The mass was composed principally of a heterogeneously enhancing solid portion and exhibited some cystic changes. Clinically and radiologically, the mass appeared to be a gastrointestinal stromal tumor of the stomach. The patient underwent exploratory laparotomy and mass excision. The surgical specimen revealed a solid firm mass measuring $13 \times 13 \times 6 \mathrm{~cm}$. Histopathological and immunohistochemical analyses identified an MPNST. The patient underwent adjuvant chemotherapy but developed local recurrence with peritoneal seeding 8 months after surgery. Despite treatment with a different chemotherapeutic regimen, the disease progressed systemically and the patient died 3 years and 5 months after surgery. The uncommon tumor location and CT findings of our present MPNST case provide valuable information in terms of future clinical diagnoses of this rare but highly malignant disease.
\end{abstract}

Keywords: Malignant Peripheral Nerve Sheath Tumor, Lesser Sac, Gastrointestinal Stromal Tumor, Retroperitoneum, Computed Tomography

\section{Introduction}

A malignant peripheral nerve sheath tumor (MPNST) is a very rare tumor, with an incidence of approximately 1:100,000 people per year (1). The term MPNST was coined in 2002 by the world health organization to replace previous heterogeneous names, such as "malignant schwannoma", "malignant neurilemmoma", "neurogenic sarcoma", and "neurofibrosarcoma" (2). The neoplasm arises from a peripheral nerve and exhibits variable differentiation toward one of the cellular components of the nerve sheath (3). The tumor is a rare form of soft tissue sarcoma, constituting $3-10 \%$ of all sarcomas (1). Approximately 50 - 60\% of MPNSTs develop in patients with neurofibromatosis type 1 (NF1) (4). MPNSTs behave aggressively, and the risks of local recurrence and metastasis are significant. The 5-year survival rate is 30 - 50\% despite application of multidisciplinary therapy (5).

Patients are usually diagnosed at 20 - 50 years of age. Only approximately $10 \%$ present at less than 20 years of age (6). MPNST most commonly affects the extremities and pelvis (1). Retroperitoneal involvement is rare. Herein, we present a case of a 16-year-old girl with a massive MPNST in the lesser sac. This case was diagnosed clinically and radiologically as a gastrointestinal stromal tumor (GIST) of the stomach, but pathologically, the tumor was diagnosed as an MPNST after surgical resection. The patient lacked any clinical evidence of NF1.

\section{Case Report}

A16-year-old female presented to the Hallym university sacred heart hospital in April 2012 with dyspepsia and abdominal distension that had progressed during the prior 6 months. She had been taking medications to treat active tuberculosis and iron-deficiency anemia. Physical examination revealed a palpable mass in the epigastric area. The laboratory data were within normal ranges, except for a hemoglobin level of $10.2 \mathrm{~g} / \mathrm{dL}$ (normal, $12-16 \mathrm{~g} / \mathrm{dL}$ ). This study was approved by the institutional review board of the Hallym university sacred heart hospital, which waived written informed consent because this case report is a retrospective review of medical records.

Contrast-enhanced abdominopelvic computed tomography (CT) demonstrated a massive well-defined mass approximately $12 \mathrm{~cm}$ in diameter in the lesser sac. Most 
of the mass was composed of heterogeneously enhancing solid material and some cystic change was shown. The mass compressed the lesser curvature of the stomach anterolaterally, displaced the pancreas and splenic vein inferiorly, and abutted the left kidney posteriorly (Figure 1). Neither lymph node enlargement nor any peritoneal seeding nodule was observed. CT findings most likely suggested that the tumor was a GIST arising from the stomach. The serum levels of carbohydrate antigen 19 - 9 (CA19-9) and carcinoembryonic antigen were $73.08 \mathrm{U} / \mathrm{mL}$ (normal, 0 - $27 \mathrm{U} / \mathrm{mL}$ ) and $1.26 \mathrm{ng} / \mathrm{mL}$ (normal, 0 - $5 \mathrm{ng} / \mathrm{mL}$ ), respectively.

The patient underwent exploratory laparotomy and mass excision. The large mass was adherent to the lesser curvature and posterior aspect of the stomach and to the superior margin of the pancreas and splenic vein. The surgical specimen revealed a solid firm mass measuring $13 \times$ $13 \times 6 \mathrm{~cm}$. The external surface of the mass was generally smooth but focally irregular and adhered to the adjacent soft tissue. The cut surface of the mass was pinkish gray and whitish, trabeculated, and hemorrhagic. No definite necrosis was evident in the mass (Figure 2A). Microscopic examination (Figure $2 \mathrm{~B}$ - E) demonstrated that the tumor consisted of spindle cells arranged in intersecting fascicles and densely cellular areas alternating with less cellular areas. Nuclear palisading was observed adjacent to the cellular fascicles. The high-power view revealed dense fascicles of spindle cells with bulky, ovoid, and irregular cellular nuclei. Elongated wavy-to-buckled nuclei were evident in the less cellular areas. Immunohistochemically, the tumor was focally positive for S-100 protein (Figure 2F) but negative for c-kit, alpha-smooth muscle actin, desmin, CK, and CD34. Fluorescence in-situ hybridization for the synovial sarcoma translocated gene was negative. These histopathological and immunohistochemical findings indicated that the tumor was an MPNST (3). The resection margin was microscopically positive.

Postoperative abdominopelvic CT and [18F]fluorodeoxyglucose positron emission tomography-CT detected no residual tumor. The serum CA19-9 level decreased to $14.7 \mathrm{U} / \mathrm{mL}$ after surgery. The patient received adjuvant chemotherapy (four cycles of doxorubicin/ifosfamide). However, follow-up CT in December 2012 revealed local tumor recurrence (a 6.3-cm-diameter mass) in the lesser sac and peritoneal seeding. Two cycles of chemotherapy (etoposide/ifosfamide/cisplatin) were administered, but follow-up CT in February 2013 revealed progression of the peritoneal seeding and a newly developed liver metastasis. After receiving one more cycle of chemotherapy (gemcitabine/docetaxel), the patient was lost to follow-up. She expired on September 2015.

\section{Discussion}

An MPNST arising in the abdomen is very rare, and to the best of our knowledge, only a few cases of retroperitoneal MPNSTs have been reported $(7,8)$. Various neoplastic diseases develop in the lesser sac. They usually mimic gastric or pancreatic tumors. As the tumor of our case was large, its origin was difficult to define; either the stomach or lesser omentum was a possibility. A massive, well-circumscribed mass exhibiting heterogeneous enhancement and some cystic changes was apparent on enhanced CT scans. Similarly, a malignant gastric GIST usually presents as a large, well-circumscribed heterogeneous mass with a central region of low density (9). Based on the CT findings and location of the mass, we assumed that the tumor was a GIST arising from the lesser curvature of the stomach or an extra-GIST of the lesser omentum. The absence of any NF1 signs contributed to no suspicion of MPNST. Therefore, we did not conduct a preoperative biopsy; the fragility of GISTs and the resultant risks of hemorrhage and dissemination preclude preoperative biopsy (10). The mass was pathologically identified as an MPNST after surgery.

Retroperitoneal MPNST has been reported to develop in the paraspinal region, close to a nerve trunk (11). The CT features of an MPNST include a large heterogeneously enhancing mass with an irregular or infiltrative margin, invasion of adjacent organs or soft tissues, and bone destruction $(11,12)$. The CT findings of our case were different except for the large heterogeneously enhancing mass. Both the unusual tumor location and CT findings of our present case of MPNST provide valuable information for future clinical diagnoses.

MPNST is typically associated with a poor outcome compared with those of other soft tissue sarcomas. The recurrence rate is as high as $40 \%$, and the most common metastatic sites are the lungs and the bone (3). The 5year survival rate ranges from 30 to $50 \%$ (5). The negative prognostic factors for MPNST are similar to those for other soft tissue sarcomas and include the tumor site (head, neck, and trunk vs. the extremities), a large tumor size, high-grade, and positive surgical margins (1). Some reports suggested that NF1 patients with MPNST experience poorer survival than do sporadic MPNST patients (13) although this has not been a consistent finding (14). Tumor size, tumor site, and microscopically incomplete resection explain the poor outcome of our present patient. Her younger age may also have influenced her unfavorable outcome (13).

Complete surgical resection is the mainstay of MPNST treatment. Incomplete surgical resection increases the risk of MPNST-specific death nearly six-fold (4). The tumor 

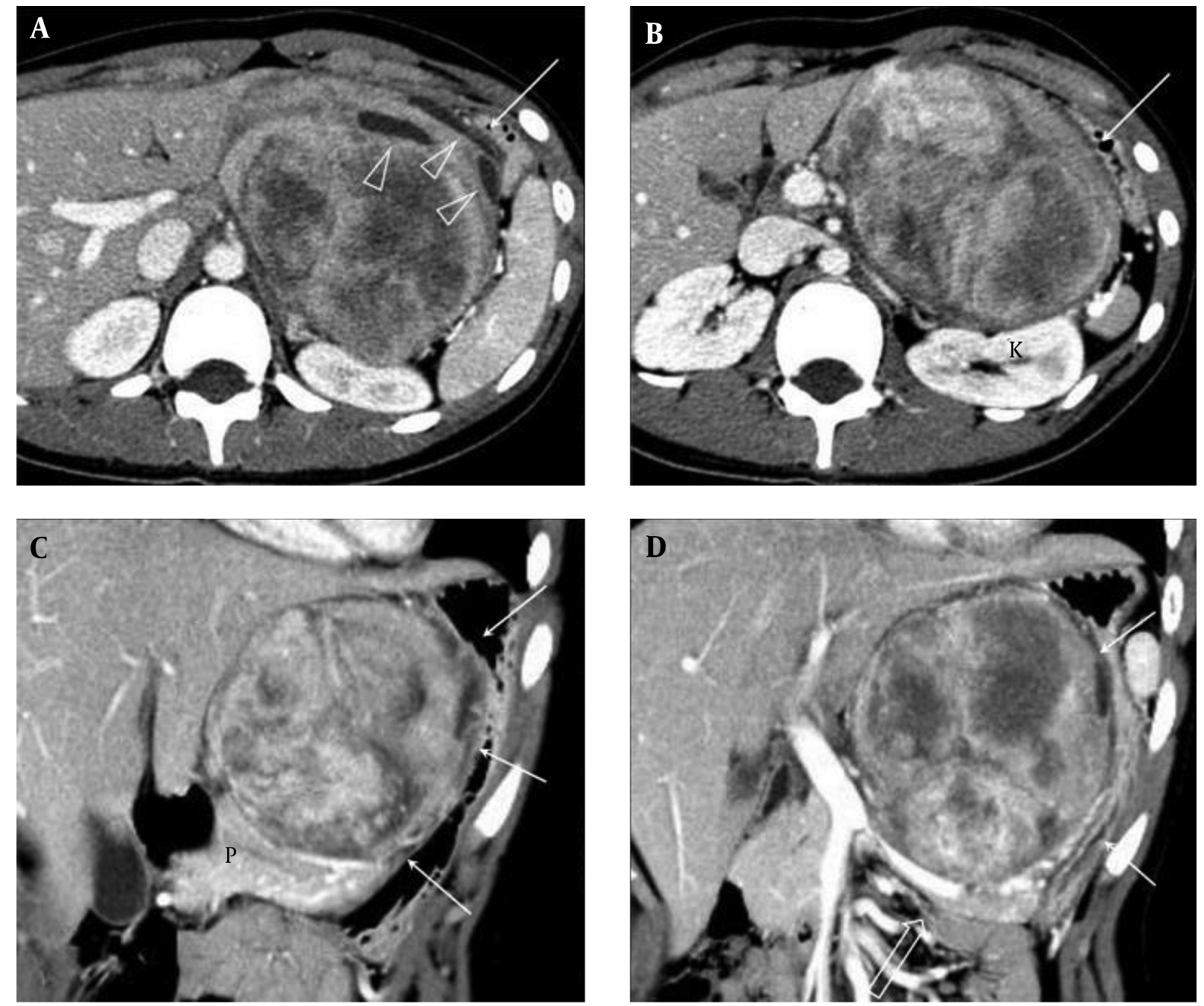

Figure 1. Computed tomography of the abdomen in a 16-year-old girl with dyspepsia and abdominal distension. A, B, Axial images show approximately $12 \mathrm{~cm}$, large, welldefined mass in the lesser sac. The mass shows heterogeneous enhancement with some low-density areas and cystic changes (arrowheads). The mass is displacing the stomach (arrows) anterolaterally and the kidney (k) posteriorly. C, D, Coronal reformatted images show that the mass compresses the lesser curvature of the stomach (arrows) and displaces the pancreas (p) and splenic vein (open arrow) inferiorly.

location in our present case adversely affected creating an adequate surgical margin (15). Adjuvant radiotherapy may improve local tumor control; however, any evidence that survival is prolonged by radiotherapy is limited (1618). If the preoperative diagnosis had been correct (identification of MPNST with a biopsy), neoadjuvant radiotherapy may have reduced the tumor volume, rendering complete excision possible (19). Role of chemotherapy for MPNST was proved more in pediatric patients than in adults. The overall response rate to primary chemotherapy was reported as $45 \%$ in a group of pediatric MPNST patients (6). First-line chemotherapy typically consists of a combination of ifosfamide and doxorubicin (1). In our present case, the MPNST recurred and progressed systematically despite the use of these chemotherapeutic agents. Research on the molecular pathway involved in MPNST pathogenesis and the development of effective target agents would aid in treatment of this very aggressive malignancy.

In conclusion, MPNSTs remain diagnostically and therapeutically challenging. This case report improves our understanding of this extremely rare but highly malignant disease. 

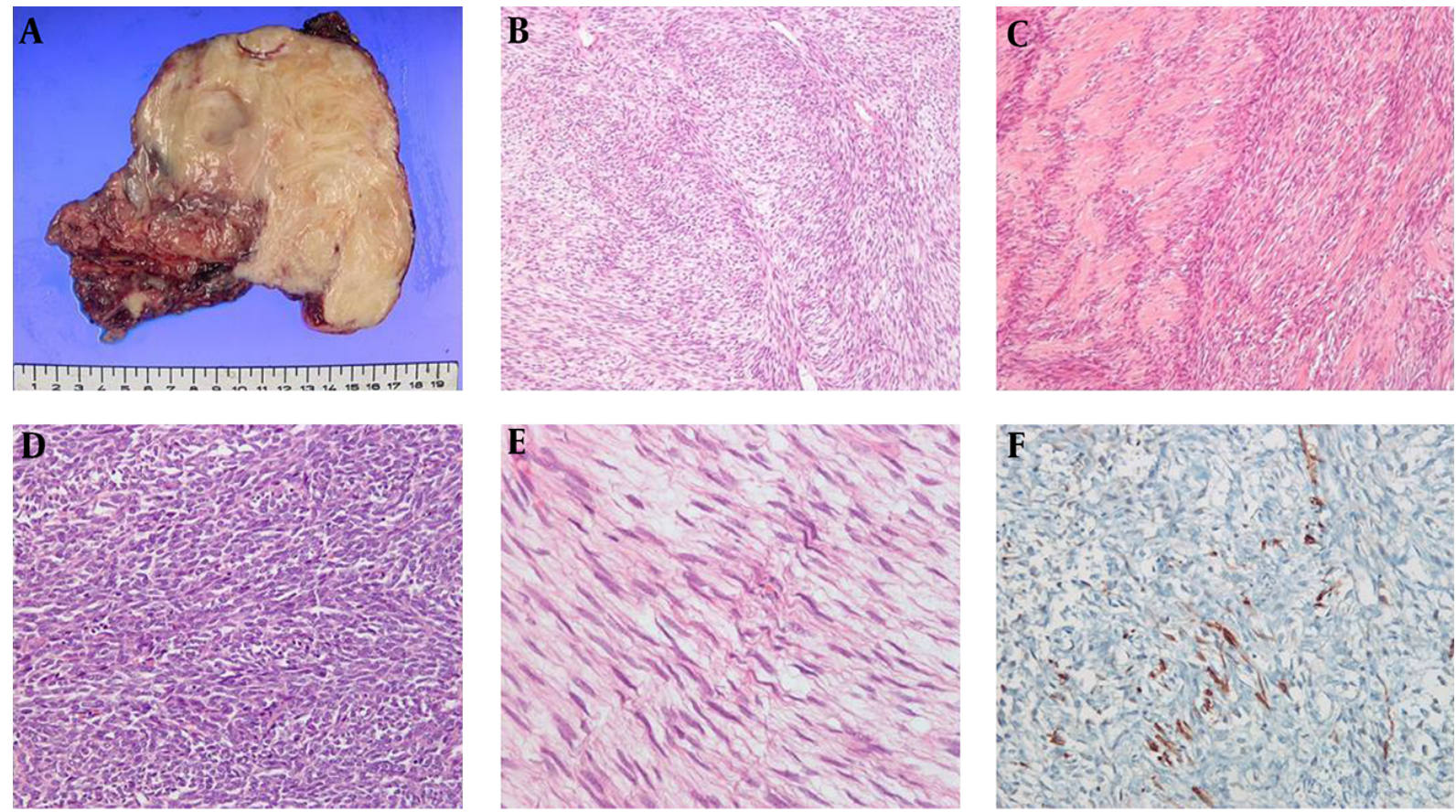

Figure 2. The surgical pathology. A, Macroscopically, the tumor is a whitish-gray oval, firm solid mass measuring $13 \times 13 \times 6 \mathrm{~cm}$, the external surface of the mass is mostly smooth but focally irregular and adherent to soft tissue; B, Microscopically spindle tumor cells are arranged in intersecting fascicles with alternating densely cellular areas and less cellular areas; C, Nuclear is evident adjacent to the cellular fascicles; D, The high-power view reveals dense fascicles of spindle cells with bulky, ovoid, and irregular cellular nuclei; E, Elongated wavy-to-buckled nuclei are shown in the less cellular areas; F, Immunohistochemically, the tumor is focally positive for S-100 protein.

\section{Footnotes}

Authors' Contributions: Seung-Gu Yeo and Eun Seok Kim contributed equally to this study. Seung-Gu Yeo and Eun Seok Kim wrote the manuscript. Min-Jeong Kim developed study concept and design, and supervised the study. Hong Il Ha, Kwanseop Lee, and Mi Jung Kwon contributed to the acquisition, analysis and interpretation of data.

\section{Financial Disclosure: None declared.}

Funding/Support: The present study was supported by the Soonchunhyang university research fund

\section{References}

1. Grobmyer SR, Reith JD, Shahlaee A, Bush CH, Hochwald SN. Malignant Peripheral Nerve Sheath Tumor: molecular pathogenesis and current management considerations.J Surg Oncol. 2008;97(4):340-9. doi: 10.1002/jso.20971. [PubMed: 18286466].

2. Perrin RG, Guha A. Malignant peripheral nerve sheath tumors. Neurosurg Clin N Am. 2004;15(2):203-16. doi: 10.1016/j.nec.2004.02.004. [PubMed: 15177319].

3. Thway K, Fisher C. Malignant peripheral nerve sheath tumor: pathology and genetics. Ann Diagn Pathol. 2014;18(2):109-16. doi: 10.1016/j.anndiagpath.2013.10.007. [PubMed: 24418643].

4. Zou C, Smith KD, Liu J, Lahat G, Myers S, Wang WL, et al. Clinical, pathological, and molecular variables predictive of malignant peripheral nerve sheath tumor outcome. Ann Surg. 2009;249(6):1014-22. doi: 10.1097/SLA.ob013e3181a77e9a. [PubMed: 19474676].
5. Fan Q, Yang J, Wang G. Clinical and molecular prognostic predictors of malignant peripheral nerve sheath tumor. Clin Transl Oncol. 2014;16(2):191-9. doi: 10.1007/s12094-013-1061-x. [PubMed: 23749326].

6. Carli M, Ferrari A, Mattke A, Zanetti I, Casanova M, Bisogno G, et al. Pediatric malignant peripheral nerve sheath tumor: the Italian and German soft tissue sarcoma cooperative group. J Clin Oncol. 2005;23(33):8422-30. doi: 10.1200/JCO.2005.01.4886. [PubMed: 16293873].

7. Alavi S, Arzanian MT, Nilipour Y. Retroperitoneal malignant peripheral nerve sheath tumor replacing an absent kidney in a child. Case Rep Oncol Med. 2013;2013:627472. doi: 10.1155/2013/627472. [PubMed: 24392233].

8. Yan B, Meng X, Shi B, Shi J, Qin Z, Wei P. A retroperitoneal NF1-independent malignant peripheral nerve sheath tumor with elevated serum CA125: case report and discussion. I Neurooncol. 2012;109(1):205-11. doi: 10.1007/s11060-012-0865-9. [PubMed: 22528792].

9. Burkill GJ, Badran M, Al-Muderis O, Meirion Thomas J, Judson IR, Fisher C, et al. Malignant gastrointestinal stromal tumor: distribution, imaging features, and pattern of metastatic spread. Radiology. 2003;226(2):527-32. doi: 10.1148/radiol.2262011880. [PubMed: 12563150].

10. Everett M, Gutman H. Surgical management of gastrointestinal stromal tumors: analysis of outcome with respect to surgical margins and technique.J Surg Oncol. 2008;98(8):588-93. doi:10.1002/jso.21030. [PubMed: 19072850].

11. Levy AD, Patel N, Dow N, Abbott RM, Miettinen M, Sobin LH. From the archives of the AFIP: abdominal neoplasms in patients with neurofibromatosis type 1: radiologic-pathologic correlation. Radiographics. 2005;25(2):455-80. doi: 10.1148/rg.252045176. [PubMed: 15798063]. 
12. Lang N, Liu XG, Yuan HS. Malignant peripheral nerve sheath tumor in spine: imaging manifestations. Clin Imaging. 2012;36(3):209-15. doi: 10.1016/j.clinimag.2011.08.015. [PubMed: 22542380].

13. Anghileri M, Miceli R, Fiore M, Mariani L, Ferrari A, Mussi C, et al. Malignant peripheral nerve sheath tumors: prognostic factors and survival in a series of patients treated at a single institution. Cancer. 2006;107(5):1065-74. doi:10.1002/cncr.22098. [PubMed: 16881077].

14. Cashen DV, Parisien RC, Raskin K, Hornicek FJ, Gebhardt MC, Mankin HJ. Survival data for patients with malignant schwannoma. Clin Orthop Relat Res. 2004(426):69-73. [PubMed: 15346054].

15. Mastoraki A, Toska F, Tsiverdis I, Kyriazi M, Tsagkas A, Danias N, et al. Retroperitoneal schwannomas: dilemmas in diagnostic approach and therapeutic management. J Gastrointest Cancer. 2013;44(4):371-4 doi: 10.1007/s12029-013-9510-x. [PubMed: 23749630].

16. Kar M, Deo SV, Shukla NK, Malik A, DattaGupta S, Mohanti BK, et al. Malignant peripheral nerve sheath tumors(MPNST)-clinicopathological study and treatment outcome of twenty-four cases. World JSurg Oncol. 2006;4:55. doi: 10.1186/1477-7819-4-55. [PubMed:16923196].

17. Stucky CC, Johnson KN, Gray RJ, Pockaj BA, Ocal IT, Rose PS, et al. Malignant peripheral nerve sheath tumors (MPNST): the Mayo Clinic experience. Ann Surg Oncol. 2012;19(3):878-85. doi: 10.1245/s10434-011-19787. [PubMed: 21861229].

18. Chung K, Han Y, Kim J, Ahn SH, Ju SG, Jung SH, et al. The first private-hospital based proton therapy center in Korea; status of the Proton Therapy Center at Samsung Medical Center. Radiat Oncol J. 2015;33(4):337-43. doi: 10.3857/roj.2015.33.4.337. [PubMed: 26756034].

19. Cuneo KC, Riedel RF, Dodd LG, Harpole DH, Kirsch DG. Pathologic complete response of a malignant peripheral nerve sheath tumor in the lung treated with neoadjuvant Ifosfamide and radiation therapy. $J$ Clin Oncol. 2012;30(28):291-3. doi: 10.1200/JCO.2012.42.8797. [PubMed: 22869889]. 